\title{
On the difference between the eccentric connectivity index and eccentric distance sum of graphs
}

\author{
Yaser Alizadeh $^{a} \quad$ Sandi Klavžar ${ }^{b}$ \\ ${ }^{a}$ Department of Mathematics, Hakim Sabzevari University, Sabzevar, Iran \\ e-mail: y.alizadeh.s@gmail.com \\ ${ }^{b}$ Faculty of Mathematics and Physics, University of Ljubljana, Slovenia \\ e-mail: sandi.klavzar@fmf.uni-lj.si
}

\begin{abstract}
The eccentric connectivity index of a graph $G$ is $\xi^{c}(G)=\sum_{v \in V(G)} \varepsilon(v) \operatorname{deg}(v)$, and the eccentric distance sum is $\xi^{d}(G)=\sum_{v \in V(G)} \varepsilon(v) D(v)$, where $\varepsilon(v)$ is the eccentricity of $v$, and $D(v)$ the sum of distances between $v$ and the other vertices. A lower and an upper bound on $\xi^{d}(G)-\xi^{c}(G)$ is given for an arbitrary graph $G$. Regular graphs with diameter at most 2 and joins of cocktail-party graphs with complete graphs form the graphs that attain the two equalities, respectively. Sharp lower and upper bounds on $\xi^{d}(T)-\xi^{c}(T)$ are given for arbitrary trees. Sharp lower and upper bounds on $\xi^{d}(G)+\xi^{c}(G)$ for arbitrary graphs $G$ are also given, and a sharp lower bound on $\xi^{d}(G)$ for graphs $G$ with a given radius is proved.
\end{abstract}

Key words: eccentricity; eccentric connectivity index; eccentric distance sum; tree AMS Subj. Class (2020): 05C12, 05C09, 05C92

\section{Introduction}

In this paper we consider simple and connected graphs. If $G=(V(G), E(G))$ is a graph and $u, v \in V(G)$, then the distance $d_{G}(u, v)$ between $u$ and $v$ is the number of edges on a shortest $u, v$-path. The eccentricity of a vertex and its total distance are distance properties of central interest in (chemical) graph theory; they are defined as follows. The eccentricity $\varepsilon_{G}(v)$ of a vertex $v$ is the distance between $v$ and a farthest vertex from $v$, and the total distance $D_{G}(v)$ of $v$ is the sum of distances between $v$ and the other vertices of $G$. Even more fundamental property of a vertex in (chemical) graph theory is its degree (or valence in chemistry), denoted by $\operatorname{deg}_{G}(v)$. (We may skip the 
index $G$ in the above notations when $G$ is clear.) Multiplicatively combining two out of these three basic invariants naturally leads to the eccentric connectivity index $\xi^{c}(G)$, the eccentric distance sum $\xi^{d}(G)$, and the degree distance $D D(G)$, defined as follows:

$$
\begin{aligned}
\xi^{c}(G) & =\sum_{v \in V(G)} \varepsilon(v) \operatorname{deg}(v) . \\
\xi^{d}(G) & =\sum_{v \in V(G)} \varepsilon(v) D(v) . \\
D D(G) & =\sum_{v \in V(G)} \operatorname{deg}(v) D(v) .
\end{aligned}
$$

$\xi^{c}$ was introduced by Sharma, Goswami, and Madan [17, $\xi^{d}$ by Gupta, Singh, and Madan [7, and $D D$ by Dobrynin and Kochetova [6] and by Gutman [8]. These three topological indices are well investigated, selected contrubutions to the eccentric connectivity index are [10, 13, 22], to the eccentric distance sum [1, 14, 21, and to the degree distance [15, 18, 19]. The three invariants were also compared to other invariants, cf. [2, 3, 4, 5, 23]. For information on additional topological indices based on eccentricity see [16].

In [11] the eccentric distance sum and the degree distance are compared, while in 24] the difference between the eccentric connectivity index and the (not defined here) connective eccentricity index is studied. The primary motivation for the present paper, however, are the papers [12, 25] in which $\xi^{d}(G)-\xi^{c}(G)$ was investigated. In [25], Zhang, $\mathrm{Li}$, and $\mathrm{Xu}$, besides other results on the two indices, determined sharp upper and lower bounds on $\xi^{d}(G)-\xi^{c}(G)$ for graphs $G$ of given order and diameter 2. Parallel results were also derived for sub-classes of diameter 2 graphs with specified one of the minimum degree, the connectivity, the edge-connectivity, and the independence number. Hua, Wang, and Wang [12] extended the last result to general graphs. More precisely, they characterized the graphs that attain the minimum value of $\xi^{d}(G)-\xi^{c}(G)$ among all connected graphs $G$ of given independence number. They also proved a related result for connected graphs with given matching number.

In this paper we continue the investigation along the lines of [12, 25] and proceed as follows. In the rest of this section definitions and some observations needed are listed. In Section 2, we give a lower and an upper bound on $\xi^{d}(G)-\xi^{c}(G)$ and in both cases characterize the equality case. The upper bound involves the Wiener index, the first Zagreb index, as well as the degree distance of $G$. In Section 3 we focus on trees and first prove that among all trees $T$ with given order and diameter, $\xi^{d}(T)-\xi^{c}(T)$ is minimized on caterpillars. Using this result we give a lower bound on $\xi^{d}(T)-\xi^{c}(T)$ for all trees $T$ with given order, the bound being sharp precisely on stars. We also give a sharp upper bound on $\xi^{d}(T)-\xi^{c}(T)$ for trees $T$ with given order. In the last section we give a sharp lower bound and a sharp upper bound on $\xi^{d}(G)+\xi^{c}(G)$, compare $\xi^{d}(G)$ with $\xi^{c}(G)$ for graphs $G$ with not too large maximum degree, and give a sharp lower bound on $\xi^{d}(G)$ for graphs $G$ with a given radius. 


\subsection{Preliminaries}

The order and the size of a graph $G$ will be denoted by $n(G)$ and $m(G)$, respectively. The star of order $n \geq 2$ is denoted by $S_{n}$; in other words, $S_{n}=K_{1, n-1}$. If $n \geq 2$, then the cocktail party graph $C P_{2 n}$ is the graph obtained from $K_{2 n}$ by removing a perfect matching. The join $G \oplus H$ of graphs $G$ and $H$ is the graph obtained from the disjoint union of $G$ and $H$ by connecting by an edge every vertex of $G$ with every vertex of $H$. The maximum degree of a vertex of $G$ is denoted by $\Delta(G)$. A graph $G$ is regular if all vertices have the same degree. The first Zagreb index [9] $M_{1}(G)$ of $G$ is the sum of the squares of the degrees of the vertices of $G$. The Wiener index [20] $W(G)$ of $G$ is the sum of distances between all pairs of vertices in $G$.

The diameter $\operatorname{diam}(G)$ and the radius $\operatorname{rad}(G)$ of a graph $G$ are the maximum and the minimum vertex eccentricity in $G$, respectively. A graph $G$ is self-centered if all vertices have the same eccentricity. It this eccentricity is $d$, we further say that $G$ is $d$-self-centered. The eccentricity $\varepsilon(G)$ of $G$ is

$$
\varepsilon(G)=\sum_{v \in V(G)} \varepsilon(v) .
$$

The eccentric connectivity index of $G$ can be equivalently written as

$$
\xi^{c}(G)=\sum_{u v \in E(G)} \varepsilon(u)+\varepsilon(v),
$$

and the eccentric distance sum as

$$
\xi^{d}(G)=\sum_{\{u, v\} \subseteq V(G)}(\varepsilon(u)+\varepsilon(v)) d(u, v) .
$$

\section{The difference on general graphs}

In this section we give some sharp upper and lower bounds on $\xi^{d}(G)-\xi^{c}(G)$ for an arbitrary graph $G$. The bounds are in terms of the eccentricity, the Wiener index, the first Zagreb index, the degree distance, the maximum degree, the size, and the order of $G$.

Theorem 2.1 If $G$ is a connected graph, then the following hold.

(i) $\xi^{d}(G)-\xi^{c}(G) \geq 2(n(G)-1-\Delta(G)) \varepsilon(G)$. Moreover, the equality holds if and only if $G$ is a regular graph with $\operatorname{diam}(G) \leq 2$.

(ii) $\xi^{d}(G)-\xi^{c}(G) \leq 2 n(G)(W(G)-m(G))+M_{1}(G)-D D(G)$. Moreover, the equality holds if and only if $G \in\left\{P_{4}\right\} \cup\left\{C P_{2 k} \oplus K_{n(G)-2 k}: 0 \leq k \leq n / 2\right\}$. 
Proof. (i) Let $v$ be a vertex of $G$. If $w$ is not adjacent to $v$, then $d(v, w) \geq 2$ and consequently $D(v)-\operatorname{deg}(v) \geq 2(n(G)-1-\Delta(G))$. Thus:

$$
\begin{aligned}
\xi^{d}(G)-\xi^{c}(G) & =\sum_{v \in V(G)} \varepsilon(v)(D(G)-\operatorname{deg}(v)) \\
& \geq \sum_{v \in V(G)} 2 \varepsilon(v)(n(G)-1-\Delta(G)) \\
& =2 \varepsilon(G)(n(G)-1-\Delta(G)) .
\end{aligned}
$$

The equality holds if and only if $D(v)-\operatorname{deg}(v)=2(n(G)-1-\Delta(G))$ for every vertex $v$. As the last equality in particular holds for a vertex of maximum degree, we infer that $G$ must be regular. Then the condition $D(v)-\operatorname{deg}(v)=2(n(G)-1-\Delta(G))$ simplifies to

$$
D(v)+\Delta(G)=2 n(G)-2 .
$$

Suppose that $\operatorname{diam}(G)=d$, and let $x_{i}, i \in\{2, \ldots, d\}$, be the number of vertices at distance $i$ from $v$. Then $n(G)=1+\Delta(G)+x_{2}+\cdots+x_{d}$ and $D(v)=\Delta(G)+2 x_{2}+$ $\cdots+d x_{d}$. Plugging these equalities into (3) yields

$$
2 \Delta(G)+2 x_{2}+\cdots+d x_{d}=2+2 \Delta(G)+2 x_{2}+\cdots+2 x_{d}-2
$$

which implies that $x_{3}=\cdots=x_{d}=0$, that is, $\operatorname{diam}(G)=2$. Finally, if $\operatorname{diam}(G)=2$, then $D(v)=\Delta(G)+2(n(G)-\Delta(G)-1)$, so (3) is fulfilled for every regular graph of diameter 2. Clearly, (3) is also fulfilled for graphs of diameter 1, that is, complete graphs.

(ii) If $v \in V(G)$, then clearly $\varepsilon(v) \leq n(G)-\operatorname{deg}(v)$. Then we deduce that

$$
\begin{aligned}
\xi^{d}(G)-\xi^{c}(G)= & \sum_{v \in V(G)} \varepsilon(v)(D(v)-\operatorname{deg}(v)) \\
\leq & \sum_{v \in V(G)}(n(G)-\operatorname{deg}(v))(D(v)-\operatorname{deg}(v)) \\
= & n(G) \sum_{v \in V(G)}(D(v)-\operatorname{deg}(v))+\sum_{v \in V(G)} \operatorname{deg}(v)^{2} \\
& -\sum_{v \in V(G)} \operatorname{deg}(v) D(v) \\
= & 2 n(G)(W(G)-m(G))+M_{1}(G)-D D(G) .
\end{aligned}
$$

The equality in the above computation holds if and only if $\varepsilon(v)=n(G)-\operatorname{deg}(v)$ holds for all $v \in V(G)$. So suppose that $G$ is a graph for which $\varepsilon(v)=n(G)-\operatorname{deg}(v)$ holds for all $v \in V(G)$ and distinguish the following two cases.

Suppose first that $\operatorname{diam}(G) \geq 3$. Let $P$ be a diametral path in $G$ and let $v$ and $v^{\prime}$ be its endpoints. Since $\varepsilon(v)=n(G)-\operatorname{deg}(v)$ and $|V(P) \backslash N[v]|=\varepsilon(v)-1$, it follows 
that $n(G)=1+\operatorname{deg}(v)+|V(P) \backslash N[v]|$. The latter means that $V(G)=N[v] \cup V(P)$. Since $\operatorname{diam}(G)=\varepsilon(v) \geq 3$ it follows that $\operatorname{deg}\left(v^{\prime}\right)=1$. Since we have also assumed that $\varepsilon\left(v^{\prime}\right)=n(G)-\operatorname{deg}\left(v^{\prime}\right)$ holds we see that $\varepsilon\left(v^{\prime}\right)=n(G)-1$ which in turn implies that $G$ is a path. Among the paths $P_{n}, n \geq 4$, the path $P_{4}$ is the unique one which fulfills the condition $\varepsilon(v)=n-\operatorname{deg}(v)$ for all $v \in V\left(P_{n}\right)$.

Suppose second that $\operatorname{diam}(G) \leq 2$. Then $\varepsilon(v) \in\{1,2\}$ for every $v \in(G)$. Since $\varepsilon(v)=n(G)-\operatorname{deg}(v)$ it follows that $\operatorname{deg}(v) \in\{n(G)-1, n(G)-2\}$. Let $V_{1}=\{v$ : $\operatorname{deg}(v)=n(G)-1\}$ and $V_{2}=\{v: \operatorname{deg}(v)=n(G)-2\}$. Then $V(G)=V_{1} \cup V_{2}$. Clearly, the subgraph of $G$ induced by $V_{1}$ is complete, and there are all possible edges between $V_{1}$ and $V_{2}$. Moreover, the complement of the subgraph of $G$ induced by $V_{2}$ is a disjoint union of copies of $K_{2}$, which means that $V_{2}$ induces a cocktail party graph. In summary, $G$ must be of the form $C P_{2 k} \oplus K_{n(G)-2 k}$, where $0 \leq k \leq n / 2$. On the other hand, the condition $\varepsilon(v)=n(G)-\operatorname{deg}(v)$ clearly holds for each vertex of $C P_{2 k} \oplus K_{n(G)-2 k}$, hence these graphs together with $P_{4}$ from the previous case are precisely the graphs that attain the equality.

\section{The difference on trees}

In this section we turn our attention to $\xi^{d}(T)-\xi^{c}(T)$ for trees $T$, and in particular on extremal trees regarding this difference.

Theorem 3.1 Among all trees $T$ with given order and diameter, $\min \left\{\xi^{d}(T)-\xi^{c}(T)\right\}$ is achieved on caterpillars.

Proof. Fix the order and diameter of trees to be considered. Let $T$ be an arbitrary tree that is not a caterpillar with this fixed order and diameter. Let $P$ be a diametral path of $T$ connecting $x$ to $y$. Then the eccentricity of each vertex $w$ of $T$ is equal to $\max \{d(w, x), d(w, y)\}$. Let $z \neq x, y$ be a vertex of $P$ and let $T_{z}$ be a maximal subtree of $T$ which contains $z$ but no other vertex of $P$. We may assume that $z$ can be selected such that $\varepsilon_{T_{z}}(z)=k \geq 2$, for otherwise $T$ is a caterpillar. Let $u$ be vertex of $T_{z}$ with $d(u, z)=k-1$ and let $v$ be the neighbor of $u$ with $d(v, z)=k-2$. Let $S=N(u) \backslash\{v\}$ and let $s=|S|$. Note that $s>0$. Let now $T^{\prime}$ be the tree obtained from $T$ by replacing the edges between $u$ and the vertices of $S$ with the edges between $v$ and the vertices of $S$.

Claim A: $\xi^{d}(T)-\xi^{c}(T)>\xi^{d}\left(T^{\prime}\right)-\xi^{c}\left(T^{\prime}\right)$.

Set $X_{d}=\xi^{d}(T)-\xi^{d}\left(T^{\prime}\right)$ and $X_{c}=\xi^{c}(T)-\xi^{c}\left(T^{\prime}\right)$. To prove the claim it is equivalent to show that $X_{d}-X_{c}>0$.

For a vertex $w \in V(G) \backslash(S \cup\{u\})$ we have $D_{T^{\prime}}(w)=D_{T}(w)-s$ and $\varepsilon_{T^{\prime}}(w) \leq \varepsilon_{T}(w)$. Moreover if $w \in S$, then $\varepsilon_{T^{\prime}}(w)=\varepsilon_{T}(w)-1$ and $D_{T}(w)=D_{T^{\prime}}(w)+n-s-2$. With 
these facts in hand we can compute as follows.

$$
\begin{aligned}
X_{d}= & \sum_{w \in V(T)} \varepsilon_{T}(w) D_{T}(w)-\sum_{w \in V\left(T^{\prime}\right)} \varepsilon_{T^{\prime}}(w) D_{T^{\prime}}(w) \\
= & \varepsilon_{T}(u) D_{T}(u)-\varepsilon_{T^{\prime}}(u) D_{T^{\prime}}(u)+\varepsilon_{T}(v) D_{T}(v)-\varepsilon_{T^{\prime}}(v) D_{T^{\prime}}(v) \\
& +\sum_{w \in S} \varepsilon_{T}(w) D_{T}(w)-\varepsilon_{T^{\prime}}(w) D_{T^{\prime}}(w) \\
& +\sum_{w \in V(T)-(S \cup\{u, v\})} \varepsilon_{T}(w) D_{T}(w)-\varepsilon_{T^{\prime}}(w) D_{T^{\prime}}(w) \\
\geq & s\left(\varepsilon_{T}(v)-\varepsilon_{T}(u)\right)+\sum_{w \in V(T)-(S \cup\{u, v\})} \varepsilon_{T}(w) s \\
& +\sum_{w \in S}\left(\varepsilon_{T}(w) D_{T}(w)-\left(\varepsilon_{T}(w)-1\right)\left(D_{T}(w)-n+2+s\right)\right) \\
= & -s+\sum_{w \in V(T)-(S \cup\{u, v\})} \varepsilon_{T}(w) s \\
& +\sum_{w \in S}\left(\left(D_{T}(w)-n+2+s\right)-\varepsilon_{T}(w)(-n+2+s)\right) \\
= & -s+\sum_{w \in V(T) \backslash(S \cup\{u, v\})} \varepsilon_{T}(w) s+(n-s-2) \sum_{w \in S} \varepsilon_{T}(w)-1+D_{T}(w) \\
= & -s+\sum_{w \in V(T) \backslash(S \cup\{u, v\})} \varepsilon_{T}(w) s \\
& +s(n-s-2) \varepsilon_{T}(u)+s\left(D_{T}(u)+n-2\right) \\
& \left.\left.s(T)-\varepsilon_{T}(u)(s+2)-s+1+(n-s-2) \varepsilon_{T}(u)+D_{T}(u)+n-3\right)\right] .
\end{aligned}
$$

Similarly, but shorter, we get that $X_{c}=2 s$. Thus

$$
\begin{aligned}
X_{d}-X_{c} \geq & s\left[\varepsilon(T)-\varepsilon_{T}(u)(s+2)\right. \\
& \left.\left.+(n-s-2) \varepsilon_{T}(u)+D_{T}(u)+n-s-4\right)\right] \\
& >0
\end{aligned}
$$

This proves Claim A. If $T^{\prime}$ is not a caterpillar, we can repeat the construction as many times as required to arrive at a caterpillar. Since at each step the value of $\xi^{d}-\xi^{c}$ is decreased, the minimum of this difference is indeed achieved on caterpillars.

Theorem 3.2 If $T$ is a tree of order $n \geq 3$, then

$$
\xi^{d}(T)-\xi^{c}(T) \geq 4 n^{2}-12 n+8 .
$$

Moreover, equality holds if and only if $T=S_{n}$. 
Proof. Let $n \geq 3$ be a fixed integer. By Theorem 3.1, it suffices to consider caterpillars. More precisely, let $T$ be a caterpillar of order $n$ and with $\operatorname{diam}(T)=d \geq 3$. Then we wish to prove that $\xi^{d}(T)-\xi^{c}(T)>\xi^{d}\left(S_{n}\right)-\xi^{c}\left(S_{n}\right)=4 n^{2}-12 n+8$. The latter equality is straightforward to check, for the strict inequality we proceed as follows.

Let $w, z \in V(T)$ be two adjacent vertices of eccentricities $d-1$ and $d-2$, respectively. Let $S=N(w) \backslash\{z\}$ and set $s=|S|$. As $\varepsilon(w)=d-1$, we have $s \geq 1$. Let further $S_{1}=V(G) \backslash(S \cup\{w, z\})$. Construct now a tree $T^{\prime}$ from $T$ by replacing the edges between $w$ and the vertices of $S$ with the edges between $z$ and the vertices of $S$. Note that $\operatorname{deg}_{T}(w)=\operatorname{deg}_{T^{\prime}}(w)+s=1+s$ and $\operatorname{deg}_{T}(z)=\operatorname{deg}_{T^{\prime}}(z)-s$, while the other vertices have the same degree in $T$ and $T^{\prime}$. Further, it is straightforward to verify the following relations:

$$
\begin{aligned}
& D_{T}(w)=D_{T^{\prime}}(w)-s, \quad \varepsilon_{T}(w)=\varepsilon_{T^{\prime}}(w) ; \\
& D_{T}(z)=D_{T^{\prime}}(z)+s, \quad \varepsilon_{T^{\prime}}(z) \leq \varepsilon_{T}(z) \leq \varepsilon_{T^{\prime}}(z)+1 ; \\
& D_{T}(x)=D_{T^{\prime}}(x)+n-s-2, \quad \varepsilon_{T}(x)=\varepsilon_{T^{\prime}}(x)+1(x \in S) ; \\
& D_{T}(y)=D_{T^{\prime}}(y)+s, \quad \varepsilon_{T^{\prime}}(y) \leq \varepsilon_{T}(y) \leq \varepsilon_{T^{\prime}}(y)+1\left(y \in S_{1}\right) .
\end{aligned}
$$

Setting $X_{d}=\xi^{d}(T)-\xi^{d}\left(T^{\prime}\right)$ we have:

$$
\begin{aligned}
X_{d}= & \sum_{v \in\{w, z\}} D_{T}(v) \varepsilon_{T}(v)-D_{T^{\prime}}(v) \varepsilon_{T^{\prime}}(v)+\sum_{v \in S} D_{T}(v) \varepsilon_{T}(v)-D_{T^{\prime}}(v) \varepsilon_{T^{\prime}}(v) \\
& +\sum_{v \in S_{1}} D_{T}(v) \varepsilon_{T}(v)-D_{T^{\prime}}(v) \varepsilon_{T^{\prime}}(v) \\
\geq & s\left(\varepsilon_{T^{\prime}}(z)-\varepsilon_{T}(w)\right)+\sum_{v \in S} D_{T}(v) \varepsilon_{T}(v)-\left(D_{T}(v)-(n-s-2)\right)\left(\varepsilon_{T}(v)-1\right) \\
& +\sum_{v \in S_{1}} D_{T}(v) \varepsilon_{T}(v)-\left(D_{T}(v)-s\right) \varepsilon_{T}(v) \\
\geq & -s+(n-s-2) \sum_{v \in S} \varepsilon_{T}(v)+\sum_{v \in S} D_{T}(v)-s(n-s-2)+s \sum_{v \in S_{1}} \varepsilon_{T}(v) \\
\geq & -s+3 s(n-s-2)+s(2(n-s-2)+2 s+1)-s(n-s-2) \\
& +3 s(n-s-2) \\
= & 5 s(n-s-3)+2 s(n-1) .
\end{aligned}
$$


Similarly, setting $X_{c}=\xi^{c}(T)-\xi^{c}\left(T^{\prime}\right)$, we have

$$
\begin{aligned}
X_{c}= & \sum_{v \in\{w, z\}}\left(\operatorname{deg}_{T}(v) \varepsilon_{T}(v)-\operatorname{deg}_{T^{\prime}}(v) \varepsilon_{T^{\prime}}(v)\right) \\
& +\sum_{v \in S}\left(\operatorname{deg}_{T}(v) \varepsilon_{T}(v)-\operatorname{deg}_{T^{\prime}}(v) \varepsilon_{T^{\prime}}(v)\right) \\
& +\sum_{v \in S_{1}}\left(\operatorname{deg}_{T}(v) \varepsilon_{T}(v)-\operatorname{deg}_{T^{\prime}}(v) \varepsilon_{T^{\prime}}(v)\right) \\
\leq & s \varepsilon_{T}(w)+\operatorname{deg}_{T}(z) \varepsilon_{T}(z)-\left(\operatorname{deg}_{T}(z)+s\right)\left(\varepsilon_{T}(z)-1\right) \\
& +s+\sum_{v \in S_{1}} \operatorname{deg}_{T}(v) \varepsilon_{T}(v)-\operatorname{deg}_{T}(v)\left(\varepsilon_{T}(v)-1\right) \\
= & 2 s+\operatorname{deg}_{T}(z)+\sum_{v \in S_{1}} \operatorname{deg}(v) \\
= & 2 n-3 .
\end{aligned}
$$

Therefore,

$$
X_{d}-X_{c} \geq(5 s(n-s-3)+2 s(n-1))-(2 n-3)>0,
$$

that is, $\xi^{d}(T)-\xi^{c}(T)>\xi^{d}\left(T^{\prime}\right)-\xi^{c}\left(T^{\prime}\right)$. Repeating the above transformation until $S_{n}$ is constructed finishes the argument.

To bound the difference $\xi^{d}(T)-\xi^{c}(T)$ for an arbitrary tree $T$ from above, we first recall the following result.

Lemma 3.3 [14, Theorem 2.1] Let $w$ be a vertex of graph G. For non-negative integers $p$ and $q$, let $G(p, q)$ denotes the graph obtained from $G$ by attaching to vertex $w$ pendant paths $P=w v_{1} \cdots v_{p}$ and $Q=w u_{1} \cdots u_{q}$ of lengths $p$ and $q$, respectively. Let $G(p+$ $q, 0)=G(p, q)-w u_{1}+v_{p} u_{1}$. If $r=\varepsilon_{G}(w)$ and $r \geq p \geq q \geq 1$, then

$$
\begin{aligned}
\xi^{d}(G(p+q, 0))-\xi^{d}(G(p, q)) \geq & \frac{p q}{6}\left[6 D_{G}(w)+p(2 p-3)+q(2 q-3)+3 p q-12 r\right. \\
& \left.+6 n(G)(p+q+r+1)+6 \sum_{v \in V(G)} \varepsilon(v)\right] .
\end{aligned}
$$

Lemma 3.4 Let $G, p, q, G(p, q)$, and $G(p+q, 0)$ be as in Lemma 3.3. Then

$$
\xi^{c}(G(p+q, 0))-\xi^{c}(G(p, q)) \leq q(3 p+2 m(G)-1)
$$


Proof. Let $\operatorname{deg}^{\prime}(v)$ and $\varepsilon^{\prime}(v)($ resp. $\operatorname{deg}(v)$ and $\varepsilon(v))$ denote the degree and the eccentricity of $v$ in $G(p+q, 0)$ (resp. $G(p, q))$. Then we have:

$$
\begin{aligned}
\operatorname{deg}^{\prime}(w) & =\operatorname{deg}(w)-1, \quad \varepsilon^{\prime}(w) \leq \varepsilon(w)+q ; \\
\operatorname{deg}^{\prime}\left(v_{i}\right) & =\operatorname{deg}\left(v_{i}\right), i \in[p-1], \quad \operatorname{deg}^{\prime}\left(v_{p}\right)=\operatorname{deg}\left(v_{p}\right)+1 ; \\
\varepsilon^{\prime}\left(v_{i}\right) & \leq \varepsilon\left(v_{i}\right)+q, i \in[p] ; \\
\operatorname{deg}^{\prime}\left(u_{j}\right) & =\operatorname{deg}\left(u_{j}\right), \quad \varepsilon^{\prime}\left(u_{j}\right)=\varepsilon\left(u_{j}\right)+p ; \\
\varepsilon^{\prime}(x) & \leq \varepsilon(x)+q, x \in V(G) .
\end{aligned}
$$

Moreover, the degrees of vertices in $G(p+q, 0)$ do not decrease. Calculating the difference of contributions of vertices in $\xi^{c}$ for $G(p+q, 0)$ and $G(p, q)$, we can estimate the difference $X_{c}=\xi^{c}(G(p+q, 0))-\xi^{c}(G(p, q))$ as follows:

$$
\begin{aligned}
X_{c} \leq & \sum_{w \neq x \in V(G)} \operatorname{deg}(x) q+\sum_{i=1}^{q} \operatorname{deg}\left(u_{i}\right) p+\sum_{i=1}^{\left\lfloor\frac{p}{2}\right\rfloor} \operatorname{deg}\left(v_{i}\right) q \\
& +\varepsilon\left(v_{p}\right)+(\operatorname{deg}(w)-1)(r+q)-\operatorname{deg}(w) r \\
= & (2 m(G)-\operatorname{deg}(w)) q+(2 q-1) p+p q+p+q(\operatorname{deg}(w)-1) \\
= & 2 q m(G)+3 p q-q .
\end{aligned}
$$

Theorem 3.5 If $T$ is a tree of order $n$, then

$$
\xi^{d}(T)-\xi^{c}(T) \leq \begin{cases}\frac{25 n^{4}}{96}-\frac{n^{3}}{6}-\frac{89 n^{2}}{48}+\frac{19 n}{6}-\frac{45}{32} ; & n \text { odd }, \\ \frac{25 n^{4}}{96}-\frac{n^{3}}{6}-\frac{43 n^{2}}{24}+\frac{19 n}{6}-2 ; & n \text { even } .\end{cases}
$$

Moreover, equality holds if and only if $T=P_{n}$.

Proof. The right side of the above inequality is equal to $\xi^{d}\left(P_{n}\right)-\xi^{c}\left(P_{n}\right)$. (The value of $\xi^{d}\left(P_{n}\right)$ has been determined in [14, while it is straightforward to deduce $\xi^{c}\left(P_{n}\right)$. Combining the two formulas, the polynomials from the right hand side of the inequality are obtained.) Suppose now that $T \neq P_{n}$. Then there is always a vertex $w$ of degree at least 3 such that we can apply Lemmas 3.3 and 3.4. Setting

$$
X_{d c}=\left(\xi^{d}\left(T(p+q, 0)-\xi^{c}(p+q, 0)\right)-\left(\xi^{d}\left(T(p, q)-\xi^{c}(p, q)\right)\right.\right.
$$


we have:

$$
\begin{aligned}
X_{d c} \geq & p q D_{T}(w)+\frac{p q}{6}(p(2 p-3)+q(2 q-3))+\frac{(p q)^{2}}{2}-2 p q r \\
& +p q n(T)(p+q+r+1)+p q \sum_{v \in V(T)} \varepsilon(v)-(2 q m(T)+3 p q-q) \\
= & p q\left(D_{T}(w)+\sum_{v \in V(T)} \varepsilon(v)-3\right)+\frac{p q}{6}\left(2 p^{2}-3 p+2 q^{2}-3 q+3 p q\right) \\
& +\operatorname{pqr}(n(G)-2)+q(p n(T)(p+q+r)-2 m(T)+1)>0
\end{aligned}
$$

and the result follows.

\section{Further comparison}

In this concluding section we give sharp lower and upper bounds on $\xi^{d}(G)+\xi^{c}(G)$, compare $\xi^{d}(G)$ with $\xi^{c}(G)$ for graphs $G$ with $\Delta(G) \leq \frac{2}{3}(n-1)$, and give a sharp lower bound on $\xi^{d}(G)$ for graphs $G$ with a given radius.

Theorem 4.1 If $G$ is a connected graph, then the following hold.

(i) $\xi^{d}(G)+\xi^{c}(G) \leq 2(n(G)-1) \varepsilon(G)+2 \operatorname{diam}(G)\left(W(G)+m(G)-2\left(\begin{array}{c}n(G) \\ 2\end{array}\right)\right)$.

(ii) $\xi^{d}(G)+\xi^{c}(G) \geq 2(n(G)-1) \varepsilon(G)+2 \operatorname{rad}(G)\left(W(G)+m(G)-2\left(\begin{array}{c}n(G) \\ 2\end{array}\right)\right)$.

Moreover, each of the equalities holds if and only if $G$ is a self-centered graph.

Proof. (i) Partition the pairs of vertices of $G$ into neighbors and non-neighbors, and using (11), we can compute as follows:

$$
\begin{aligned}
\xi^{d}(G)= & \sum_{\{u, v\} \subseteq V(G)}(\varepsilon(u)+\varepsilon(v)) d(u, v) \\
= & \sum_{u v \in E(G)}(\varepsilon(u)+\varepsilon(v))+2 \sum_{\substack{\{u, v\} \subseteq V(G) \\
d(u, v) \geq 2}}(\varepsilon(u)+\varepsilon(v)) \\
& +\sum_{\substack{\{u, v\} \subseteq V(G) \\
d(u, v) \geq 2}}(\varepsilon(u)+\varepsilon(v))(d(u, v)-2) \\
= & \xi^{c}(G)+\sum_{\substack{\{u, v\} \subseteq V(G)\\
}}(\varepsilon(u)+\varepsilon(v))-2 \xi^{c}(G) \\
& +\sum_{\substack{\{u, v\} \subseteq V(G) \\
d(u, v) \geq 2}}(\varepsilon(u)+\varepsilon(v))(d(u, v)-2) \\
& -\xi^{c}(G)+2(n(G)-1) \varepsilon(G) \\
& +2 \operatorname{diam}(G)\left(W(G)+m(G)-2\left(\begin{array}{c}
n(G) \\
2
\end{array}\right)\right) .
\end{aligned}
$$


The inequality above becomes equality if and only if $\varepsilon(v)=\operatorname{diam}(G)$ for every $v \in$ $V(G)$. That is, the equality holds if and only if $G$ is a self-centered graph.

(ii) This inequality as well as its equality case are proved along the same lines as (i). The only difference is that the inequality $\varepsilon(u)+\varepsilon(v) \leq 2 \operatorname{diam}(G)$ is replaced by $\varepsilon(u)+\varepsilon(v) \geq 2 \operatorname{rad}(G)$.

In our next result we give a relation between $\xi^{d}(G)$ and $\xi^{c}(G)$ for graph $G$ with maximum degree at most $\frac{2}{3}(n(G)-1)$.

Theorem 4.2 If $G$ is a graph with $\Delta(G) \leq \frac{2}{3}(n-1)$, then $\xi^{d}(G) \geq 2 \xi^{c}(G)$. Moreover, the equality holds if and only if $G$ is 2-self-centered, $\frac{2}{3}(n(G)-1)$-regular graph.

Proof. Set $n=n(G)$ and let $v$ be a vertex of $G$. Since $\operatorname{deg}(v)<n-1$ we have $\varepsilon(v) \geq 2$. Therefore $D(v) \geq 2(n-1)-\operatorname{deg}(v)$ with equality holding if and only if $\varepsilon(v)=2$. Using the assumption that $\operatorname{deg}(v) \leq \frac{2}{3}(n-1)$, equivalently, $2 n-2 \geq 3 \operatorname{deg}(v)$, we infer that $\varepsilon(v) D(v) \geq 2 \varepsilon(v) \operatorname{deg}(v)$. Summing over all vertices of $G$ the inequality is proved. Its derivation also reveals that the equality holds if and only if $\operatorname{deg}(v)=\frac{2}{3}(n-1)$ and $\varepsilon(v)=2$ for each vertex $v \in V(G)$.

To conclude the paper we give a lower bound on the eccentric distance sum in terms of the radius of a given graph. Interestingly, the cocktail-party graphs are again among the extreme graphs.

Theorem 4.3 If $G$ is a graph with $\operatorname{rad}(G)=r$, then

$$
\xi^{d}(G) \geq\left(n(G)-1+\left(\begin{array}{l}
r \\
2
\end{array}\right)\right) \varepsilon(G) .
$$

Equality holds if and only if $G$ is a complete graph or a cocktail-party graph.

Proof. Set $n=n(G)$ and let $v \in V(G)$. Let $P$ be a longest path starting in $v$. Separately considering the neighbors of $v$, the last $\varepsilon(v)-2$ vertices of $P$, and all the other vertices, we can estimate that

$$
\begin{aligned}
D(v) & \geq \operatorname{deg}(v)+(3+\cdots+\varepsilon(v))+2(n-1-\operatorname{deg}(v)-(\varepsilon(v)-2)) \\
& =2 n-\operatorname{deg}(v)+\frac{\varepsilon(v)^{2}-3 \varepsilon(v)}{2}-1 .
\end{aligned}
$$

Since $n-\operatorname{deg}(v) \geq \varepsilon(v)$ for every vertex $v \in V(G)$, we have $D(v) \geq n+\varepsilon(v)+\frac{\varepsilon(v)^{2}-3 \varepsilon(v)}{2}-$ 1. Consequently, having the fact $\varepsilon(v) \geq r$ in mind, we get $D(v) \geq n-1+\left(\begin{array}{l}r \\ 2\end{array}\right)$. Multiplying this inequality by $\varepsilon(v)$ and summing over all vertices of $G$ the claimed inequality is proved.

From the above derivation we see that the equality can holds only if $\varepsilon(v)=r=n-$ $\operatorname{deg}(v)$ holds for every $v \in V(G)$. From the equality part of the proof of Theorem 2.1(ii) we know that this implies $\operatorname{diam}(G) \leq 2$. For the equality we must also have $D(v)=$ $n-1+\left(\begin{array}{l}r \\ 2\end{array}\right)$ for every $v$. If $r=2$ this means that $D(v)=n$ and hence $\operatorname{deg}(v)=n-2$. It follows that $G$ is a cocktail-party graph. And if $r=2$, then we get a complete graph. 


\section{Acknowledgements}

Sandi Klavžar acknowledges the financial support from the Slovenian Research Agency (research core funding P1-0297 and projects J1-9109, J1-1693, N1-0095).

\section{References}

[1] S. Chen, S. Li, Y. Wu, L. Sun, Connectivity, diameter, minimal degree, independence number and the eccentric distance sum of graphs, Discrete Appl. Math. 247 (2018) 135-146.

[2] P. Dankelmann, M.J. Morgan, S. Mukwembi, H.C. Swart, On the eccentric connectivity index and Wiener index of a graph, Quaest. Math. 37 (2014) 39-47.

[3] K.Ch. Das, N. Trinajstić, Relationship between the eccentric connectivity index and Zagreb indices, Comput. Math. Appl. 62 (2011) 1758-1764.

[4] K. Ch. Das, M.J. Nadjafi-Arani, Comparison between the Szeged index and the eccentric connectivity index, Discrete Appl. Math. 186 (2015) 74-86.

[5] K.Ch. Das, G. Su, L. Xiong, Relation between degree distance and Gutman index of graphs, MATCH Commun. Math. Comput. Chem. 76 (2016) 221-232.

[6] A.A. Dobrynin, A.A. Kochetova, Degree distance of a graph: a degree analogue of the Wiener index, J. Chem. Inf. Comput. Sci. 34 (1994) 1082-1086.

[7] S. Gupta, M. Singh, A.K. Madan, Eccentric distance sum: A novel graph invariant for predicting biological and physical properties, J. Math. Anal. Appl. 275 (2002) $386-401$.

[8] I. Gutman, Selected properties of the Schultz molecular topological index, J. Chem. Inf. Comput. Sci. 34 (1994) 1087-1089.

[9] I. Gutman, N. Trinajstić, Graph theory and molecular orbitals. Total $\pi$-electron energy of alternant hydrocarbons, Chem. Phys. Lett. 17 (1972) 535-538.

[10] P. Hauweele, A. Hertz, H. Mélot, B. Ries, G. Devillez, Maximum eccentric connectivity index for graphs with given diameter, Discrete Appl. Math. 268 (2019) $102-111$.

[11] H. Hua, H. Wang, X. Hu, On eccentric distance sum and degree distance of graphs, Discrete Appl. Math. 250 (2018) 262-275.

[12] H. Hua, H. Wang, M. Wang, The difference between the eccentric distance sum and eccentric connectivity index, Ars Combin. 144 (2019) 3-12. 
[13] A. Ilić, I. Gutman, Eccentric connectivity index of chemical trees, MATCH Commun. Math. Comput. Chem. 65 (2011) 731-744.

[14] A. Ilić, G. Yu, L. Feng, On the eccentric distance sum of graph, J. Math. Anal. Appl. 381 (2011) 590-600.

[15] S. Li, Y. Song, H. Zhang, On the degree distance of unicyclic graphs with given matching number, Graphs Combin. 31 (2015) 2261-2274.

[16] A.K. Madan, H. Dureja, Eccentricity based descriptors for QSAR/QSPR. In: Novel Molecular Structure Descriptors - Theory and Applications II, I. Gutman, B. Furtula (Eds.), Univ. Kragujevac, Kragujevac (2010) 91-138.

[17] V. Sharma, R. Goswami, A.K. Madan, Eccentric connectivity index: A novel highly discriminating topological descriptor for structure - property and structure - activity studies, J. Chem. Inf. Comput. Sci. 37 (1997) 273-282.

[18] A.I. Tomescu, Unicyclic and bicyclic graphs having minimum degree distance, Discrete Appl. Math. 156 (2008) 125-130.

[19] H. Wang, L. Kang, Further properties on the degree distance of graphs, J. Comb. Optim. 31 (2016) 427-446.

[20] H. Wiener, Structural determination of paraffin boiling points, J. Amer. Chem. Soc. 69 (1947) 17-20.

[21] Y.-T. Xie, S.-J. Xu, On the maximum value of the eccentric distance sums of cubic transitive graphs, Appl. Math. Comput. 359 (2019) 194-201.

[22] K. Xu, K.C. Das, H. Liu, Some extremal results on the connective eccentricity index of graphs, J. Math. Anal. Appl. 433 (2016) 803-817.

[23] K. Xu, X. Li, Comparison between two eccentricity-based topological indices of graphs, Croat. Chem. Acta 89 (2016) 499-504.

[24] K. Xu, Y. Alizadeh, K.Ch. Das, On two eccentricity-based topological indices of graphs, Discrete Appl. Math. 233 (2017) 240-251.

[25] H. Zhang, S. Li, B. Xu, Extremal graphs of given parameters with respect to the eccentricity distance sum and the eccentric connectivity index, Discrete Appl. Math. 254 (2019) 204-221. 